\title{
Phenotypic Detection of MRSA and Inducible Clindamycin Resistance among Clinical Isolates of Staphylococcus-Study done in a Tertiary Level Hospital
}

\author{
Ravindra S. Rathore ${ }^{1}$, Usha Verma ${ }^{1}$, Shahbaz Alam Khan ${ }^{1}$, Ekta Gupta ${ }^{2}$, \\ Eshank Gupta ${ }^{2}$ and Prabhu Prakash ${ }^{1 *}$
}

${ }^{1}$ Microbiology, Dr. S.N. Medical College, Jodhpur, India

${ }^{2}$ AIIMS, Jodhpur, India

*Corresponding author

\section{Keywords}

Clindamycin,

Resistance,

MRSA,

Staphylococcus

Article Info

Accepted:

18 April 2019

Available Online:

10 May 2019

\section{A B S T R A C T}

Staphylococcus aureus is a versatile human pathogen causing infections ranging from relatively mild skin and soft tissue infection to life threatening sepsis, pneumonia and toxic shock syndrome. The increasing incidence of a variety of infections and, especially, the expanding role of community-associated methicillin-resistant $S$. aureus (MRSA)--has led to emphasis on the need for safe and effective agents to treat both systemic and localized staphylococcal infections. Clindamycin is considered to be one of the alternative agents in these infections. The present study was aimed to detect prevalence of inducible clindamycin resistance among $S$. aureus isolates and to study the relationship between clindamycin and methicillin resistance and correlation with multidrug resistance. During a period of6 months, a total of 245 Staphylococcal isolates from various clinical specimens were included in the study. Antimicrobial susceptibility test was done by Kirby-Bauer's disc diffusion method, MRSA detection was done by using Cefoxitin discs. For detection of inducible clindamycin resistance, D test was done by double disc synergy (DDS) test using erythromycin and clindamycin antibiotic discs and three different phenotypes were interpreted as methicillin-sensitive (MS) phenotype (D test negative), inducible MLSB (iMLSB) phenotype (D test positive), and constitutive MLSB phenotype. In study time of 6 months 245 non repeated isolates of Staphylococci were detected from various clinical samples like blood, urine, pus, vaginal swab and other samples, 157 were from Paediatrics and 33 from gynae. IPD and 55 were from OPD, 148 were $S$. aureus and 97 were CONS. Out of all 126 isolates were MRSA and 119 were MSS. In blood culture maximum MRSA were detected 86 out of 142(60.56\%) Staphylococcal isolates. Vancomycin (5.30\%) and Linezolid (6.93\%) showed minimum resistance while Ciprofloxacin showed highest resistance $67.75 \%$ ) Inducible Clindamycin resistance was seen in 40(16.32\%) isolates,92 (37.55\%) were having constitutional MLSB phenotype and $58(23.67 \%)$ were MS Phenotype Laboratories where Vitek2 and molecular facilities are not available phenotypic detection of MRSA and inducible Clindamycin detection using DDS in all Staphylococcal isolates can be cost effective laboratory methodology and can guide in judicious use of antibiotics. 


\section{Introduction}

Staphylococci are ubiquitous and most common cause of localized suppurative lesion in human beings. Their ability to develop resistance to penicillin and other antibiotics enhance its importance as human pathogen, especially in hospital enviournment.

S. aureus causes disease either by the production of toxins that produces tissue destruction or by direct invasion and destruction of tissue. Most $S$. aureus infections resolve spontaneously or in response to antibiotic treatment, but in recent years there has been increasing concern about the emergence of $S$. aureus strains that have developed resistance to multiple antibiotics. ${ }^{2}$

Spectrum of staphylococci varies from commensals on human skin to dreadful diseases like septicemia, sss, toxic shock syndrome. Other infections are infective endocarditis involving both native as well as prosthetic valve, wound and surgical site infection, skin and soft tissue infection, CNS infection, ocular infection, osteomyelitis, respiratory tract infection, urinary tract infection, toxic mediated syndromes, diarrhea, enterocolitis and infections in immunocompromised host. ${ }^{\mathbf{3 , 4}, 5}$

The emergence of Methicillin-resistant Staphylococcus aureus (MRSA) has posed a serious therapeutic challenge. Infected and colonized patients in hospitals mediate the dissemination of MRSA strains, and hospital staff is the main source of transmission. This leads to serious endemic and epidemic MRSA infections. ${ }^{6}$ The possible predisposing factors that increase the chance of emergence and spread of MRSA are prolonged and repeated hospitalization, indiscriminate use of antibiotics, lack of awareness, intravenous drug abuse, and presence of indwelling medical devices. ${ }^{7}$
Methicillin-resistant Staphylococcus aureus (MRSA) are increasingly being reported as multidrug resistant with high resistance to macrolides (erythromycin, clarithromycin) and lincosamides (clindamycin, lincomycin), leaving very few therapeutic options ${ }^{2}$. When resistance was first described in 1961, methicillin was used to test and treat infections caused by $S$. aureus. However, oxacillin, which is in the same class of drugs as methicillin, was chosen as the agent of choice for testing staphylococci in the early 1990s, and this was modified to include cefoxitin later. If oxacillin and cefoxitin are tested, why are the isolates called "MRSA" instead of "ORSA"? The acronym MRSA is still used by many to describe these isolates because of its historic role.

The Macrolide Lincosamide-Streptogramin B (MLSB) family of antibiotics serves as an alternative therapeutic agent to treat such $S$. aureus infections with clindamycin being the preferred agent ${ }^{3}$.However, the widespread use of MLSB antibiotics has led to an increase in the number of staphylococcal strains acquiring resistance to these antibiotics as well ${ }^{4}$.

However, one important issue in clindamycin treatment is the risk of clinical failure during therapy. Therapeutic failures caused by MLSB inducible resistance are being more commonly reported.

The MLS family of antibiotics has three different mechanisms of resistance: target site modification, enzymatic antibiotic inactivation and macrolide efflux pumps ${ }^{5}$.

Inducible MLSB resistance cannot be determined using standard susceptibility test methods, including standard broth-based or agar dilution susceptibility tests ${ }^{6}$. Low levels of erythromycin are the most effective inducer of inducible MLSB resistance ${ }^{7}$. 
Antimicrobial susceptibility data are important for the management of infections, but false susceptibility results may be obtained if isolates are not tested for inducible clindamycin resistance ${ }^{3}$. This study demonstrates a simple, reliable and significant method for characterization of Staphylococcal isolates by doing culture on selective medium Mannitol Salt Agar on which all Staphylococcusaureus produces yellow colour colonies, MRSA detection by Cefoxitin disc and detecting inducible resistance(by doubledisc diffusion test) in all clinical isolates of $S$. aureus and CONS.

The main objectives of this study to isolate coagulase positive and coagulase negative staphylococci by growth on Mannitol Salt Agar and coagulase test and phenotypic detection of MRSA and MSA among various clinical isolates by using Cefoxitin discs. To study various types of clindamycin resistance among MRSA and MSA isolates of Staphylococci. Also to study prevalence of inducible $\mathrm{MLS}_{\mathrm{B}}$ phenotype, constitutive $\mathrm{MLS}_{\mathrm{B}}$ phenotype and MS phenotype in clindamycin sensitivity and resistant profile of MRSA and MSA isolates of Staphylococci.

\section{Materials and Methods}

The present study was a prospective study conducted during a period of 6 months on the samples received in microbiology lab, Umaid hospital, Dr. S.N. Medical College, Jodhpur for culture sensitivity test. A total of 245 Staphylococci were isolated from various clinical specimens like blood culture (142), urine (43), pus and wound swab (29), vaginal swab (20), others (11) (sputum, throat swab, aspirates, body fluids, respiratory, central line/neck line/umbilical catheter tips), etc. were included in the study. Identification of Staphylococci were done by standard biochemical techniques ${ }^{6,9}$. Antimicrobial susceptibility testing was done by Kirby-
Bauer's disc diffusion method according CLSI-2017 guideline. For detection of mec AMediated methicillin(Oxacillin) resistance using cefoxitin, $30 \mu \mathrm{g}$ cefoxitin disk was placed and plates were incubated at $35^{\circ} \mathrm{C}$ for $24 \mathrm{~h}$. Isolates with zone diameters $\leq 21 \mathrm{~mm}$ were labelled as mec A positive methicillin resistant ${ }^{9}$. For detection of inducible clindamycin resistance, a disk approximation test was performed by placing a $2 \mu \mathrm{g}$ clindamycin disc from $21 \mathrm{~mm}$ away from the edge of a $15 \mu \mathrm{g}$ erythromycin disc $^{9}$. Following overnight incubation at $37^{\circ} \mathrm{C}$, three different phenotypes were appreciated and interpreted as follows-

1. Methicillin-sensitive (MS) phenotype: $S$. aureus isolates exhibiting resistance to erythromycin (zone size $\leq 13 \mathrm{~mm}$ ), while sensitive to clindamycin (zone size $\geq 21 \mathrm{~mm}$ ) and giving circular zone of inhibition around clindamycin ( $\mathrm{D}$ test negative).

2. Inducible $\mathrm{MLS}_{\mathrm{B}}$ phenotype: $\mathrm{iMLS}_{\mathrm{B}} S$. aureus isolates which showed resistance to erythromycin (zone size $\leq 13 \mathrm{~mm}$ ) while being sensitive to clindamycin (zone size $\geq 21 \mathrm{~mm}$ ) and giving $\mathrm{D}$ shaped zone of inhibition around clindamycin with flattening towards erythromycin disc (D test positive)

3. Constitutive $\mathrm{MLS}_{\mathrm{B}}$ phenotype: $\mathrm{cMLS}_{\mathrm{B}} S$. aureus isolates which showed resistance to both erythromycin (zone size $\leq 13 \mathrm{~mm}$ ) and clindamycin (zone size $\leq 14 \mathrm{~mm}$ ) with circular shape zone of inhibition around clindamycin.

\section{Results and Discussion}

In study time of 6 months 245 Staphylococcus sp. were isolated from various clinical samples received from IPD and OPD patients registered in various paediatrics and gynaecology department in Umaid Hospital Jodhpur. In study time OUT of 245 Staphylococcal isolates 55 were from OPD 
patients and 190 from IPD patients (157 were from Paediatrics and 33 from gynaeIPD). All samples were processed in microbiology lab and identification of isolates were done according to standard text book of microbiology. On the basis of growth on Mannitol Salt Agar medium and coagulase test staphylococci were further characterised as Staph aureus which produced yellow colonies on MSA and were coagulase positive and coagulase negative staph (CONS) which produced pink colonies on MSA and were coagulase negative. In study time from all clinical samples Staphylococcusaureus and CONS were $(148,97)$ isolated i.e. from blood cultures done by Bactac method 142 Staph.(86,56), from pus 29 Staph.(14,15), from urine samples 43 Staph. $(28,15)$, from vaginal swab 30 Staph.(14,16) and others sputum, E.T. tube, central line, throat swab etc. 11 Staphylococcus $(6,5)$ were isolated.

For detection of MRSA Cefoxitin antibiotic disk were used and result interpretation was done according to CLSI guidelines and in our study 126 were MRSA isolates and 119 were MSA. Prevalence of MRSA and MSA isolates were highest in blood culture $(86,56)$ followed by urine $(18,22)$ and pus $(12,17)$ etc.

Antibiotic sensitivity test was done by disk diffusion technique (Kirby- Bauer method) using Ciprofloxacin, Ampicillin, Ceftriaxone, Cloxacin, Clindamycin, Cefoxitin, Erythromycin, Gentamycin, Linezolid and Vancomycin according to CLSI guideline 2017. These showed drug resistance to commonly used antibiotics Ciprofloxacin in 67.75\%, Ampicillin and Ceftriaxone 58.77\%, Cloxacin 52.65\%, Clindamycin (40.81\%), Cefoxitin (35.91\%), Erythromycin $27.34 \%$, Gentamycin $\quad 15.51 \%$, Linezolid 6.93\%,Vancomycin $5.30 \%$ and Linezolid and Vancomycin both in $3.26 \%$.

When $\mathrm{D}$ test was performed using
Clindamycin and Erythromycin using double disk synergy (DDS)test according to CLSI guideline 2017. In 55 isolates were sensitive with both Erythromycin and Clindamycin antibiotics this was higher in MSS (42)isolates then MRSA (13). Erythromycin and clindamycin resistance was seen in 92 isolates more in MRSA(62) isolates then MSS (30) showing constitutive $\mathrm{MLS}_{\mathrm{B}}$ phenotype. Erythromycin resistance and Clindamycin sensitivity with positive $\mathrm{D}$ test was seen in 40 isolates which showed inducible $\mathrm{MLS}_{\mathrm{B}}$ phenotype. While in 58 isolates D test was negative this showed MS phenotype.

The overall percentage resistance for all three phenotypes was as follows:- Inducible clindamycin resistance 40 (16.32\%),Constitutive clindamycin resistance 92 (37.55\%), MS phenotype 58 (23.67\%). Percentage of both inducible and constitutive resistance was higher among MRSA isolates as compare to MSSA.

S. aureus is an important nosocomial and community acquired pathogen worldwide which can cause both superficial and deep pyogenic infections as well as a number of toxin mediated illnesses. ${ }^{16}$ The increasing frequency of staphylococcal infections among patients and changing patterns in antimicrobial resistance have led to renewed interest in the use of CL therapy to treat such infections Frank et al., 2002 $2^{23}$ CL is frequently used to treat skin and bone infections because of its tolerability, cost, oral form and excellent tissue penetration, and the fact that it accumulates in abscesses and no renal dosing adjustments are needed Kasten, $1999^{24}$. Clindamycin is also used as an alternative for patients who are allergic to penicillin $^{22}$. The strains carrying inducible erm gene are resistant to the inducer and remain susceptible to non-inducer macrolides and lincosamides ${ }^{23}$ Treatment of an infection caused by a strain carrying inducible erm 
gene using clindamycin or any non-inducer macrolide can lead to clinical failure ${ }^{21,22,23}$. Constitutive mutants can be selected in vitro in the presence of clindamycin or any other non-inducer macrolide as they are widespread among methicillin-resistant strains. In vitro routine tests for clindamycin susceptibility may fail to detect inducible clindamycin resistance due to erm genes resulting in treatment failure, thus necessitating the need to detect such resistance by a simple D test on a routine basis.

Out of 245 staphylococcal isolates $60.40 \%$ were coagulase positive staphylococci and $51.42 \%$ were MRSA and $48.58 \%$ were MSS isolates our result were in concordance with authors who have reported a higher prevalence of MRSA in their studies like Velvizhi et al., (2011). Fasih et al., (2010). While in studies done by authors like Deotale et al., (2010), Gadepalli et al., (2006), Yilmaz et al., (2007) and Azap et al., (2005) had shown higher prevalence of MSS isolates.

In our study drug resistance to commonly used antibiotics Ciprofloxacin in $67.75 \%$, Ampicillin and Ceftriaxone 58.77\%,Cefoxitin $52.65 \%$, Clindamycin $(40.81 \%)$, Coxacillin $35.91 \%$, Erythromycin $27.34 \%$, Gentamycin $15.51 \%$, Linezolid $6.93 \%$,Vancomycin $5.30 \%$ and Linezolid and Vancomycin both in $3.26 \%$. While study done by Tyagi (2016) et al., showed maximum resistance in $S$. aureus isolates was against Penicillin 10U (92.1\%) followed by Amoxicillin-Clavulanic acid 20/10 $\mu \mathrm{g}(47.8 \%)$ and Ciprofloxacin $5 \mu \mathrm{g}$ $(43.4 \%)$. However, all $(100 \%)$ the isolates were susceptible to Vancomycin $30 \mu \mathrm{g}$, Teicoplanin $20 \mu \mathrm{g}$ and Linezolid $30 \mu \mathrm{g}$. The resistance pattern did not vary with different phenotypes. In present study inducible Clindamycin resistance was seen in 40 (16.32\%) cases out of which 21 were MRSA and 19 were MSS which showed inducible $\mathrm{MLS}_{\mathrm{B}}$ phenotype. Constitutional $\mathrm{MLS}_{\mathrm{B}}$ phenotype were seen in 92 (37.55\%) serotypes out of which 62 were MRSA and 30 were MSS. MS phenotype were seen in 58(23.67\%) isolates 30 were MRSA and 28 were MSS. In our study in MRSA isolates percentage of inducible clindamycin resistance and MS phenotype (21 and 30 respectively) were higher as compared to MSS (19 and 28 respectively) isolates. This was in concordance with a few of the studies done by Deotale et al., [6] found inducible clindamycin resistance of $27.6 \%$ in MRSA and $1.6 \%$ in MSSA; Ajantha et al.,[7] found inducible clindamycin resistance of $21.1 \%$ in MRSA and $4.19 \%$ in MSSA; Mohamed Rahabar et al., [8] reported 22.6\% in MRSA and $4 \%$ in MSSA. In a study done by Gupta (2013) found high prevalence of Erythromycin resistance isolates 90(30\%). Among these $47(15.67 \%)$ isolates tested positive for inducible clindamycin resistance by $\mathrm{D}$ test while rest of the isolates negative for D test, out of which $11(3.67 \%)$ were shown to have constitutive clindamycin resistance and 32 (10.67\%) showed true sensitive to clindamycin (MS phenotype). It was also observed that percentage of inducible clindamycin resistance and MS phenotype were higher among MRSA $(39.45 \%$ and $23.85 \%$ respectively) as compare to MSSA $(2.09 \%$ and $3.14 \%$ respectively).

In study done by Yilmaz, the inducible CL-R phenotype level was $24.4 \%$ among MRSA isolates, $14.8 \%$ among MSSA isolates, 25.7\%among MRCNS isolates and $19.9 \%$ among MSCNS isolates.

In another study conducted in Turkey by Azap et al., 2005, 5.7\% among MRSA isolates, $3.6 \%$ of MSSA isolates, $30.8 \%$ of MRCNS isolates and $15.3 \%$ of MSCNS isolates were determined to have the inducible CL-R phenotype. 
Schreckenberger et al., (2004) reported incidences of inducible CL-R of $7-12 \%$ for MRSA, $19-20 \%$ for MSSA and $14-35 \%$ for CNS in two hospitals.

Reporting Staphylococcus aureus strains as susceptible to clindamycin without checking for inducible clindamycin resistance may result in inappropriate clindamycin therapy High prevalence of clindamycin resistance, especially c MLSB resistance, in our community shows that antimicrobial susceptibility test is essential when clindamycin is an option for therapy of $S$. aureus infection (Fig. 1-4 and Table 1).

Table.1 Distribution of Isolates

\begin{tabular}{|l|c|c|c|c|}
\hline Susceptibility pattern (Phenotype) & MRSA & MSSA & (TOTAL & Percentage (\%) \\
\hline E-S, CD-S & 13 & 42 & 55 & $(22.44)$ \\
\hline E-R, CD-R (Constitutive MLSB) & 62 & 30 & 92 & $(37.55)$ \\
\hline $\begin{array}{l}\text { E-R, CD-S, D test positive } \\
\text { (Inducible MLSB) }\end{array}$ & 21 & 19 & 40 & $(16.32)$ \\
\hline $\begin{array}{l}\text { E-R, CD-S, D test negative (MS } \\
\text { phenotype) }\end{array}$ & 30 & 28 & 58 & $(23.67)$ \\
\hline Total & 126 & 119 & & 245 \\
\hline
\end{tabular}

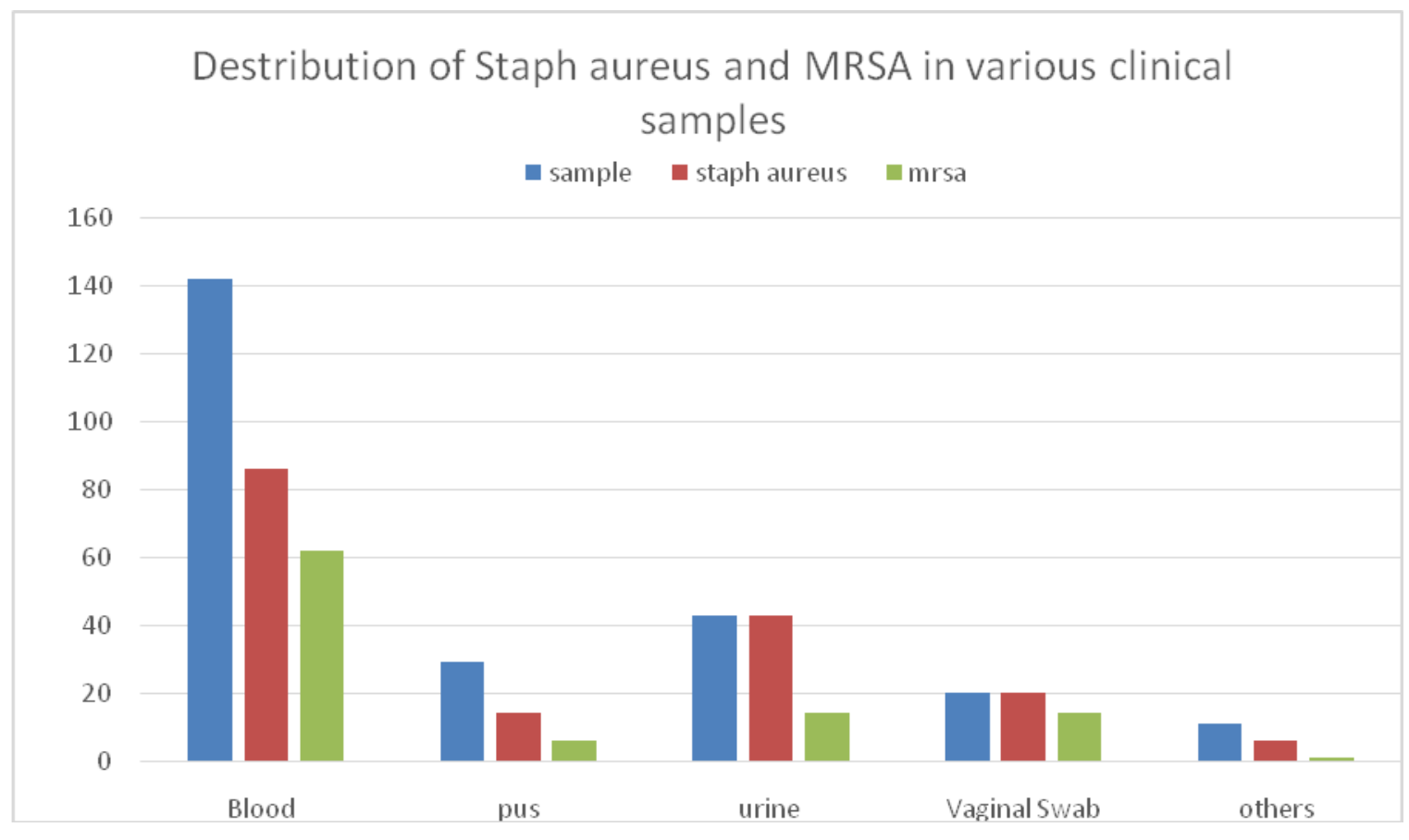




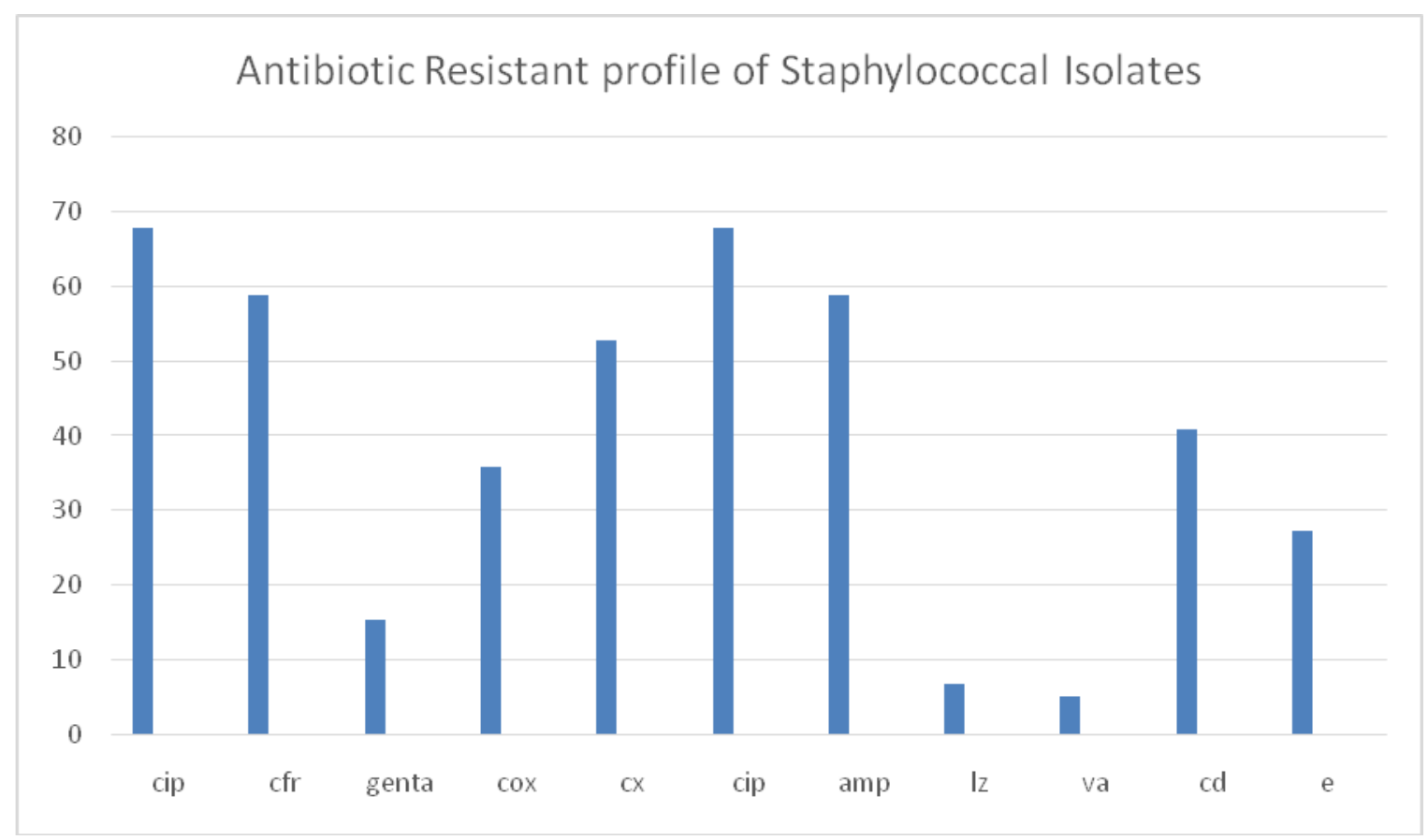

Fig.1\&2 Growth on Mannitol Salt Agar and Yellow colonies of S. aureus
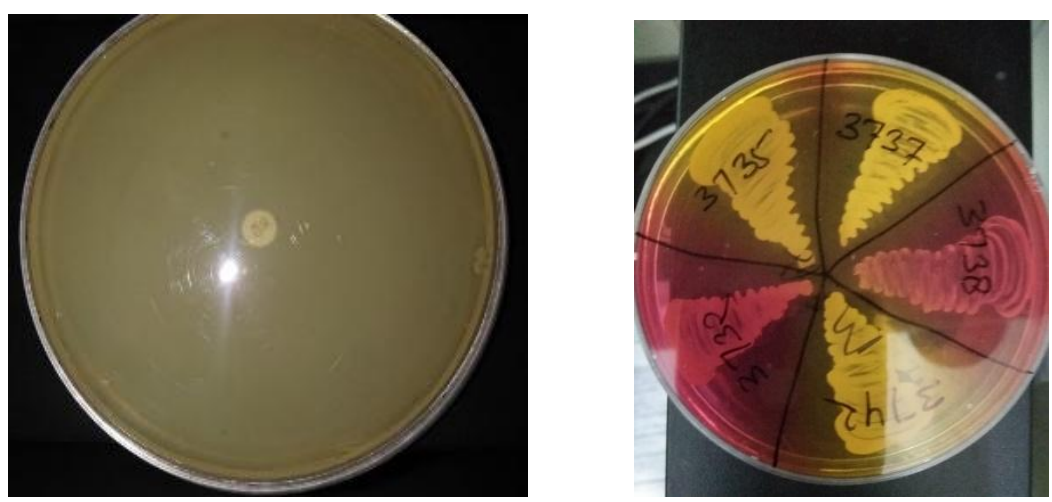

Fig.3\&4 MRSA Test using Cefoxitin and MSSA isolate
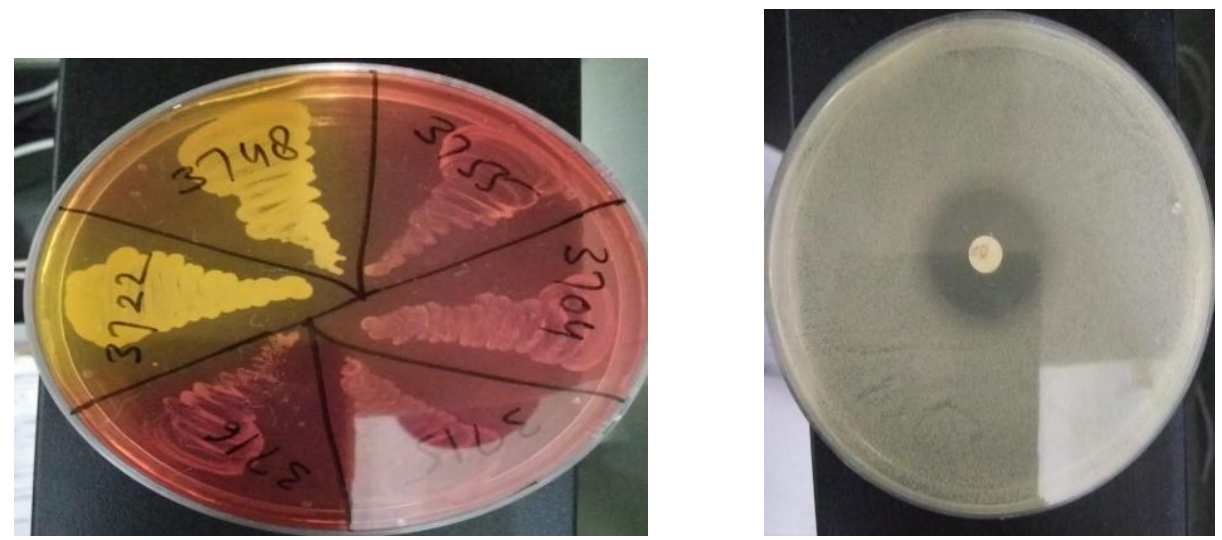
Fig.5\&6 D test positive
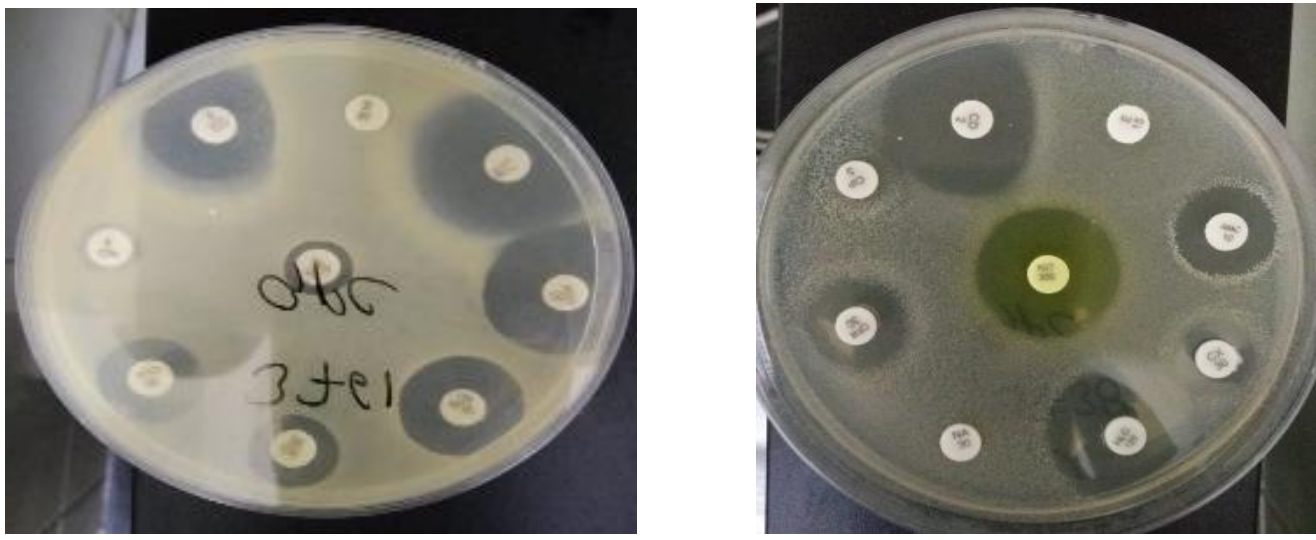

Fig.7\&8 Antibiogram of Staphylococcal isolates on MHA

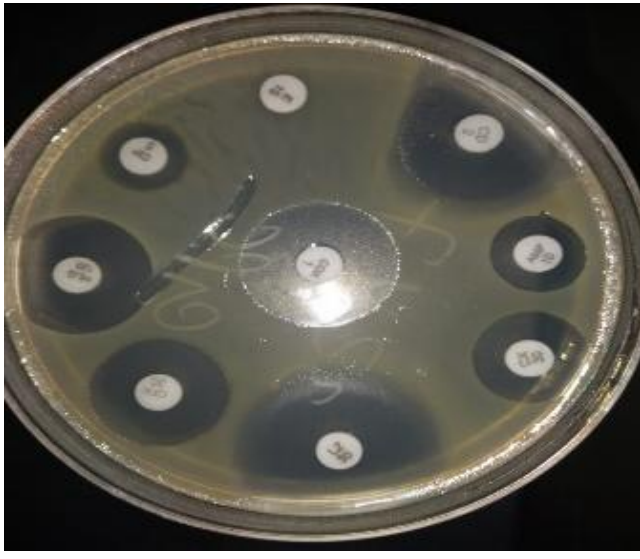

Methodology used in present study was a reliable method for detection of MRSA and inducible resistance to clindamycin in erythromycin resistant isolates of Staphylococcus aureus so that reporting of inducible clindamycin resistance in $S$. aureus can be done on routine bases. D-test can be used as a simple, auxiliary, and reliable method to delineate inducible and constitutive clindamycin resistance in routine testing so that clindamycin can be used safely and effectively in those patients with true clindamycin-susceptible strains as clindamycin is not a suitable drug for D test positive isolates; while it can definitely prove to be a drug of choice in case of $\mathrm{D}$ test negative isolates.

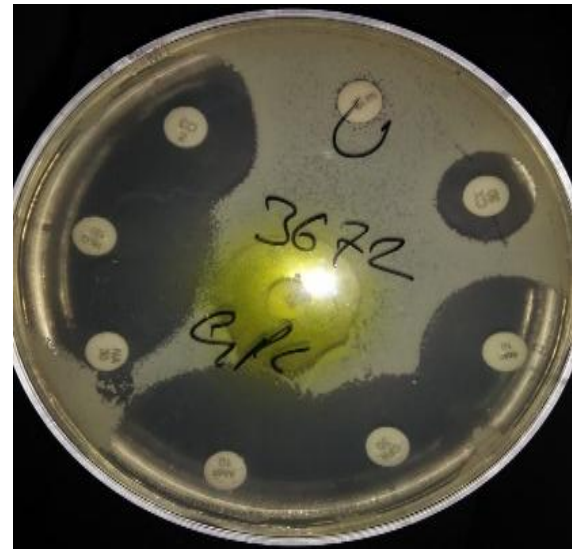

\section{References}

1. Fokas, S., Fokas, S., Tsironi, M., Kalkani, M. and Dionysopouloy, M. (2005). Prevalence of inducible clindamycin resistance in macrolide resistant Staphylococcus spp. Clin Microbiol Infect 11, 337-340.

2. Srinivasan A, Dick JD, Perl TM. Vancomycin resistance in Staphylococci. Clin Microbiol Rev 2002;15:430-8.

3. Deotale V, Mendiratta DK, Raut U, et al., Inducible clindamycin resistance in Staphylococcus aureus isolated from clinical samples. Indian J Med Microbiol 2010; 28:124-6

4. Goldman, R. C. and Capobianco, J. O. (1990). Role of an energydependent 
efflux pump in plasmid pNE24-mediated resistance to 14- and 15-membered macrolides in Staphylococcus epidermidis. Antimicrob Agents Chemother 34, 1973-1980.

5. Weisblum, B. and Demohn, V. (1969). Erythromycin-inducible resistance in Staphylococcus aureus: survey of antibiotic classes involved. J Bacteriol 98, 447-452.

6. Koneman's Color Atlas and Textbook of Diagnostic Microbiology, seventh edition.

7. Weisblum B: Erythromycin resistance by ribosome modification. Antimicrob. Agents Chemother. 1985; 39:577-585.

8. Nakajima Y: Mechanisms of bacterial resistance to macrolide antibiotics. J Infect Chemother 1999; 5: 61-74.

9. National Committee for Clinical Laboratory Standards - CLSI. Performance Standards for Antimicrobial Susceptibility Testing; Seventeen Information Supplement. M100-S17. 2007.

10. Ajantha JS, Kulkarni RD, Shetty J, Shubadha C and Jain P: Phenotypic detection of Inducible clindamycin resistance in Staphylococcus aureus isolates by using lower limit of recommended interdisk distance. Indian J Pathol Microbiol 2008; 51: 376-8.

11. Rahabar $M$ and Hajia $M$ : Inducible clindamycin resistance in Staphylococcus aureus: A cross sectional report. Pak J Biol Sci 2007;10:189-92

12. Gupta Y, Gupta G, Garg SP: phenotypic detection of inducible clindamycin resistance amongst Staphylococcal isolates. IJPRBS, 2013; Volume 2(1): 267-272

13. Yilmaz G, Aydin K, Iskender S, Rahmet Caylan and IftiharKoksal Detection and prevalence of inducible clindamycin resistance in staphylococci Journal of Medical Microbiology (2007), 56, 342345
14. Azap, O. K., Arslan, H., Timurkaynak, F., Yapar,G., Oruc, E. and Gagir, U. (2005). Incidence of inducible clindamycin resistance in staphylococci: first results from Turkey. Clin Microbiol Infect 11, 582-584.

15. Schreckenberger, P. C., Ilendo, E. and Ristow, K. L. (2004). Incidence of constitutive and inducible clindamycin resistance in Staphylococcus aureus and coagulase-negative staphylococci in a community and a tertiary care hospital. J Clin Microbiol 42, 277s7-2779.

16. Leclercq R. Mechanisms of resistance to macrolides and lincosamides: Nature of the resistance elements and their clinical implications. Clin Infect Dis 2002;34:482-92.

17. Drinkovic D, Fuller ER, Shore KP, Holland DJ, Ellis-Pegler R. Clindamycin treatment of Staphylococcus aureus expressing inducible clindamycin resistance. J Antimicrob Chemother 2001; 48: 315-6.

18. Siberry GK, Tekle T, Carroll K, Dick J. Failure of clindamycin treatment of methicillin-resistant Staphylococcus aureus expressing inducible clindamycin resistance in vitro. Clin Infect Dis 2003; 37: 1257-60.

19. Fiebelkorn KR, Crawford SA, McElmeel ML, et al., Practical disc diffusion method for detection of inducible clindamycin resistance in Staphylococcus aureus and coagulase negative staphylococci. J Clin Microbiol 2003; 41:4740-4.

20. Practical disk diffusion method for detection of inducible clindamycin resistance in Staphylococcus aureus and coagulase negative staphylococci. J Clin Microbiol 2003;41:4740-4.

21. Weisblum B, Demohn V. Erythromycin inducible resistance in Staphylococcus aureus. Survey of antibiotic classes involved. J Bacteriol1969;98:447-52. 
22. Watanakunakorn C. Clindamycin therapy of Staphylococcus aureus endocarditis. Clinical relapse and development of resistance to clindamycin, lincomycin and erythromycin. Am J Med 1976;60:41925.

23. Frank, A. L., Marcinak, J. F., Mangat, P. D., Tjhio, J. T., Kelkar, S.,
Schreckenberger, P. C. and Quinn, J. P. (2002). Clindamycin treatment of methicillin-resistant Staphylococcus aureus infections in children. Pediatr Infect Dis J 21, 530-534.

24. Kasten, M. J. (1999). Clindamycin, metronidazole, and chloramphenicol. Mayo Clin Proc 74, 825-833.

\section{How to cite this article:}

Ravindra S. Rathore, Usha Verma, Shahbaz Alam Khan, Ekta Gupta, Eshank Gupta and Prabhu Prakash. 2019. Phenotypic Detection of MRSA and Inducible Clindamycin Resistance among Clinical Isolates of Staphylococcus-Study done in a Tertiary Level Hospital. Int.J.Curr.Microbiol.App.Sci. 8(05): 2213-2222. doi: https://doi.org/10.20546/ijcmas.2019.805.261 